\title{
Anisotropy of Electronic Conduction in Oriented Poly(ethylene 2,6-naphthalate) Film
}

\author{
Seiji HAYASHI, Minoru IshiharadA, and Shogo SAITO* \\ Department of Materials Science and Technology, \\ Graduate School of Engineering Sciences, \\ Kyushu University, Kasuga, Fukuoka 816, Japan
}

(Received December 4, 1984)

\begin{abstract}
The method for measuring the anisotropy of photocurrent along three orthogonal directions in polymer films was proposed. Oriented poly(ethylene 2,6-naphthalate) films were employed as samples. Photocurrent along the fiber axis increased with drawing, whereas that along the direction normal to film planes decreased. The anisotropy of electronic conduction resulted from the orientation of crystallites was measured, for the first time, in an insulating polymer film which has no $\pi$-conjugated system of electrons in molecular chains.
\end{abstract}

KEY WORDS Poly(ethylene 2,6-naphthalate) / Photoconduction /

Electronic Conduction / Anisotropy / Orientation / Electrical Property /

Electrical properties of electron-conducting polymers have been actively investigated in recent years. Understanding of the electronic conduction in polymer films on the basis of higher-order structures is very important. The anisotropy of electrical conduction has been studied in some polymers. Seanor ${ }^{1}$ measured the electrical conductivity of doubly oriented nylon 66. In his study, however, the conduction was not due to electronic carriers but ionic carriers. As a matter of course, the anisotropy of electronic conduction in polyacetylene $^{2}$ and polydiacetylene ${ }^{3}$ has been studied in parallel and perpendicular directions to $\pi$-conjugation.

Hirota has proposed a new method using a four-electrode system for measuring the conductivity along the three orthogonal directions in insulating polymer films and examined the anisotropy of ionic conduction in doublyoriented nylon 12 film. $^{4,5}$ The measurement of the anisotropy for the electronic conduction, however, has not been tried.

\footnotetext{
* To whom correspondence should be addressed.
}

We developed the method for measuring the anisotropy of photocurrent by applying Hirota's method. As photocurrent is due to generation and transport of electronic carriers, the developed method should give information on the electronic conduction in polymer films.

In this paper, we describe the method for measuring the anisotropy of photocurrent at first. The higher-order structures in oriented poly(ethylene 2,6-naphthalate) (PEN) films were examined. The relationship between the electronic conduction and the oriented higherorder structure is discussed.

\section{EXPERIMENTAL}

PEN films employed in our study were biaxially drawn films with the crystallinity of ca. $45 \%$ (PEN-original), which were originally prepared by Teijin Ltd. The original film was uniaxially drawn again at $433 \mathrm{~K}$. These uniaxially drawn films with the draw ratio $\left(l / l_{0}\right)$ of 2.0 and 2.5 are called as PEN-2 and PEN-2.5, 
respectively. The thickness of the film was $100-120 \mu \mathrm{m}$.

The X-ray diffraction photographs were taken with a rotating anode unit (Rigaku Denki Ltd., Rotaflex RU-200). The X-ray beam ( $\mathrm{Ni}$ filtered $\mathrm{Cu}-K_{\alpha}$ ) was irradiated in a normal direction to the film surface (TV), along the edge direction (EV) and the drawing direction (End V), as is shown in Figure 3. X-Ray diffraction diagrams were obtained by using a counter technique.

The semitransparent gold electrodes were evaporated in the same configuration as reported by Hirota. The geometry factor of $a / b$ was 1.4. The film was mounted on the sample holder which was set in an electro-shielded chamber. All electrical measurements were carried out in a helium atmosphere. The light source was a $1 \mathrm{~kW}$ xenon lamp with appropriate filters. Dry batteries' were used as a DC power supply to exclude any electric noise, and the photocurrent was measured using a vibrating reed electrometer (TR-84M, Takeda Riken).

\section{METHOD OF PHOTOCURRENT MEASUREMENT}

Consideration of the following two points is indispensable for the measurement of the anisotropy of photocurrent in insulating polymer films.

1. Distribution of electric potential in the bulk of a sample.

2. Distribution of photo-generated carriers in the bulk of a sample.

The first has been analyzed mathematically by Hirota $^{3}$ and Wintle. ${ }^{6}$ The second is what we consider here. In the usual measurements of photocurrent, only one side of a sample film is irradiated. As the number of absorbed photons follows Beer's law through an optical absorbance, the distribution of absorbed photons across a sample is represented by the curve 1 in Figure 1. We can assume that the number of photo-generated carriers is pro-

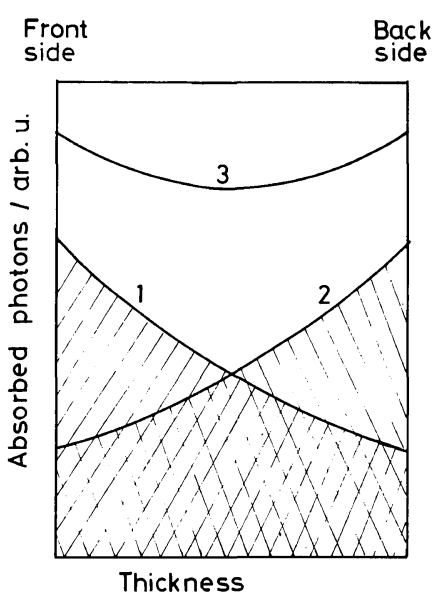

Figure 1. Distribution of absorbed photons as a function of thickness.

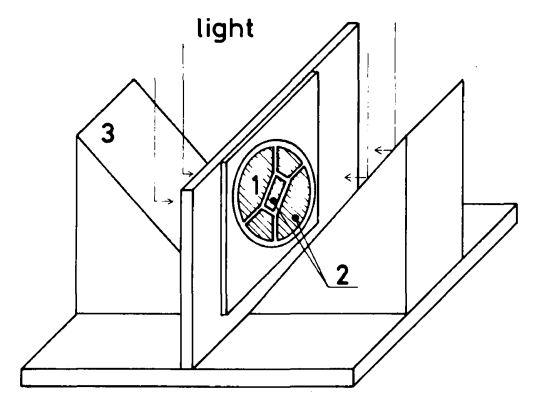

Figure 2. The sample holder: 1, sample; 2, electrodes; 3 , mirror.

portional to that of absorbed photons in a sample. Evidently, the number of carriers present in the bulk of a sample is not uniform. An appropriate correction must be made, if one attempts to compare the photocurrent parallel to a film surface with that perpendicular to a film surface. However, the distribution of absorbed photons can be made to be approximately uniform in the bulk (curve 3 in Figure 1), when both sides of a sample film are irradiated simultaneously. In this particular case, correction on the distribution of photo-generated carriers may not be necessary. In addition, one has to choose proper wavelength of light to ensure enough penetration of light into the bulk of a film. In other words, the condition of the bulk generation 
of carriers should be satisfied.

Figure 2 shows the sample holder used in this study. It has two mirrors in order to irradiate simultaneously both surfaces of a polymer film. By using this sample holder and applying Hirota's method, the evaluation of the anisotropy of photocurrent became possible. All the values obtained experimentally were corrected by the same procedure as described in the literature. ${ }^{6}$

\section{RESULTS AND DISCUSSION}

\section{(A) Higher-Order Structure of Drawn PEN} Film

Figure 3 shows the wide angle X-ray photographs of the original film, PEN-2 and PEN2.5. Sharp Debye rings were observed in the TV photograph of the original film. The characteristic pattern of TV photograph changed to the sharp spots on the layer lines with drawing as shown in the TV photograph for the PEN-2 and PEN-2.5. This meant that the fiber axis became parallel to the drawn direction. Whereas in the EV and the End V photographs the sharp orientation pattern was observed at all the samples. About twenty reflections were observed, and most of them can be indexed on the basis of the uniplanar orientation of (110) to the film surface of samples in any draw ratio. The crystallographic constants of PEN which belonged to the triclinic system were adopted from the data reported by Menčik. ${ }^{7}$

Figure 4 represents the orientation of lattices in the sample deduced from the X-ray diffraction. That is, (a) is the X-ray diffraction photograph of End V for the PEN-2.5 film, (b) is the reciprocal lattices of $(h k 0)$ plane and lattice plane indices, and (c) reveals the relationship between the unit cell and the film surface. The planes of naphthalene rings cor-

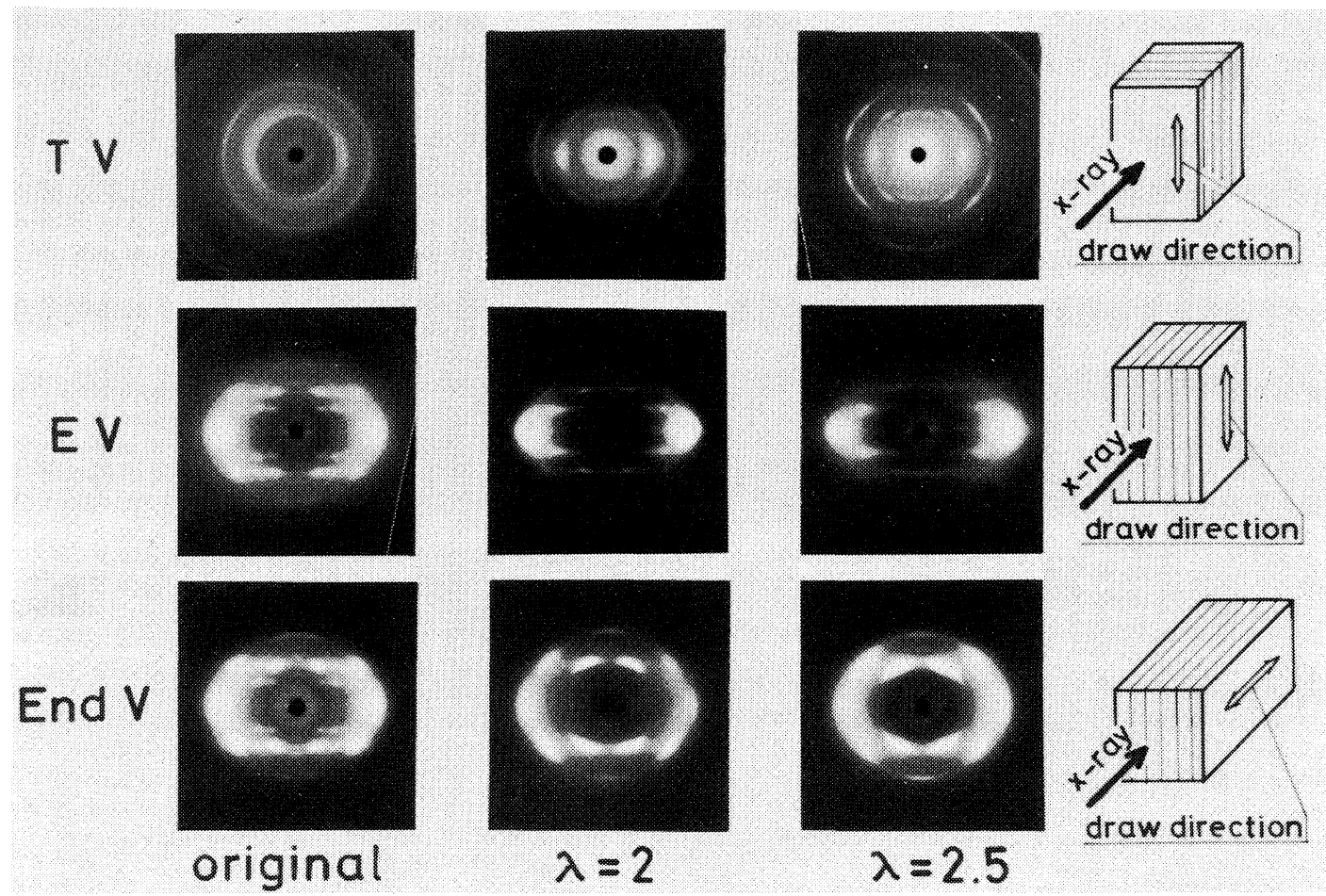

Figure 3. X-Ray photographs of PEN films with various draw ratios and the directions of incident $\mathrm{X}$ ray. 


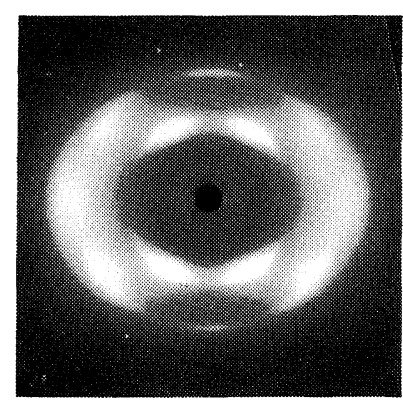

(a)

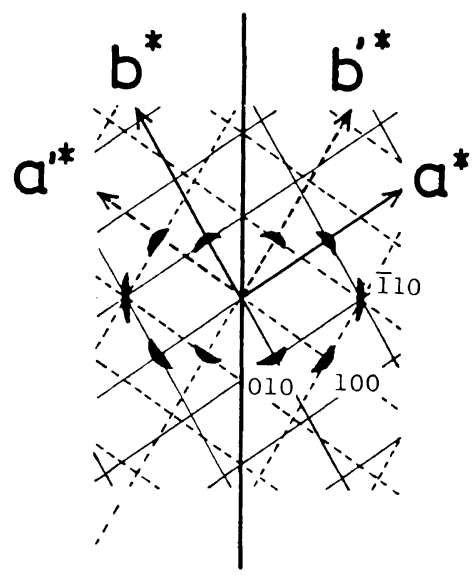

(b)

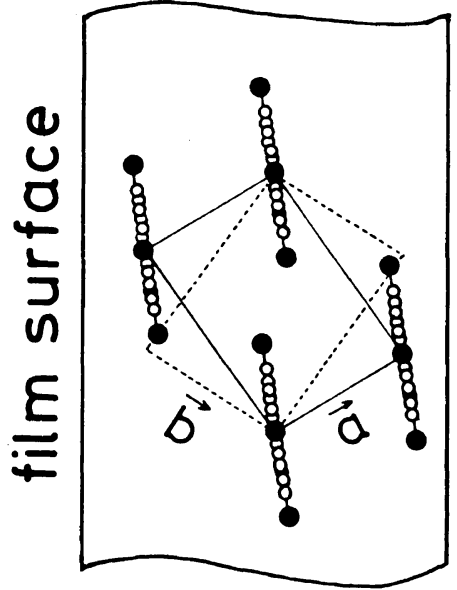

(c)

Figure 4. (a) X-Ray photographs of the End V for the PEN-2.5. (b) Reciprocal lattices of $(h k 0)$ planes and lattice plane indices. (c) Schematic representation of unit cells in PEN film.

respond to the (430) plane. $^{7}$ We can consider that the plane of naphthalene rings is approximately parallel to the film surface, because the angle between $\langle\overline{4} 30\rangle$ and $\langle\overline{1} 10\rangle$ is $7.7^{\circ}$.

The characteristic change is observed in the End V photographs in Figure 3. The sharp diffractions in the original changed gradually into the arc ones through the drawing process. The estimation of the orientation coefficients or the pole figures might be desired, if one tries to evaluate quantitatively the degree of the crystallite orientation. In the triclinic system, however, the determination of the orientation coefficients is very complicated. The intensity distribution for (110) diffraction was then measured in the azimuthal direction by a diffractometry technique. The results are shown in Figure 5. The $Z$ axis is the fiber axis and the $Y$ axis is normal to the film surface. Figure 5(b) and (c) are the intensity distribution around $X$ axis, $I(\chi)$ and that around $Z$ axis, $I(\omega)$ respectively. No change of the orientation around the $X$ axis with drawing was observed in Figure 5(b). The azimuthal breadth of the PEN-2.5 became larger with drawing (Figure 5(c)). These facts indicate that the uniplanar orientation of (110) plane in the PEN-original became disordered around the fiber axis by uniaxial stretching of the film.

Based on above results, the schematic models for the orientation of crystallites in the original and the drawn film are proposed in Figure 6, where the each rectangle represents the crystallites of the polymer. The changes of the higher-order structure are expected to have great influence on the anisotropy of photocurrents.

\section{(B) Electronic Conduction in PEN Films \\ i) Experimental Verification by Using an} Isotropic Film

The verification of the system constructed on the basic concept described in the preceding sections was considered to be necessary before performing the measurements on the PEN samples. The precision of this measuring system was proved by measuring the photocurrent parallel and perpendicular to the film surface in isotropic samples. The isotropic sample employed was poly( $N$-vinylcarbazole) trinitrofluorenone (PVK-TNF) (10:1) film. Monochromatic light of $620 \mathrm{~nm}$ was used for the generation of carriers in the bulk and that of $400 \mathrm{~nm}$ for the generation on the surface of the sample. 

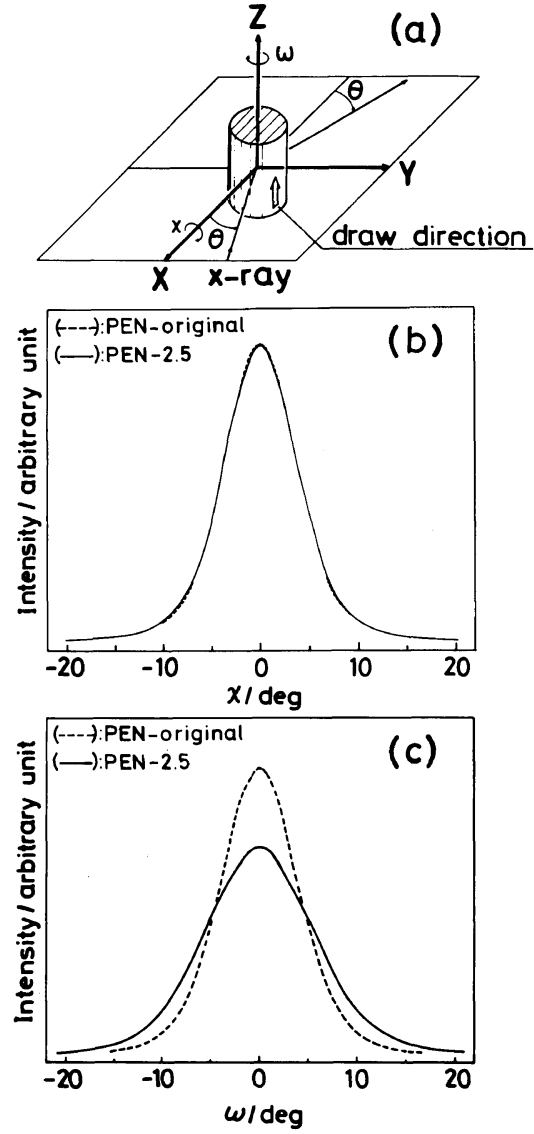

Figure 5. (a) Directions of the incident X-ray beam and rotational parameters of sample. (b) Intensity distribution for (110) reflections around $X$ axis; (c) around $Z$ axis.

Table I shows the photocurrents; $J_{x}, J_{y}, J_{z}$ along $X, Y$, and $Z$ axes at $344 \mathrm{~K}$, where $X$ and $Z$ axes are parallel to the film surface, and $Y$ axis is perpendicular to the film surface as shown in Figure 7. All the values of photocurrent were corrected with the transmittance of gold electrodes. $J_{x}$ agreed with $J_{z}$ in the both conditions of bulk and surface generation of carriers, indicating the accuracy of our system. In the case of the bulk generation of carriers, $J_{x}$ and $J_{z}$ coincided approximately with $J_{y}$, while the latter was definitely lower than the former in the case of the surface generation. Small difference between $J_{x}$ and $J_{y}$ in the bulk generation is attributable to the large $a / b$ value

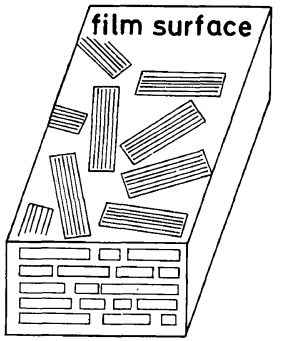

original film

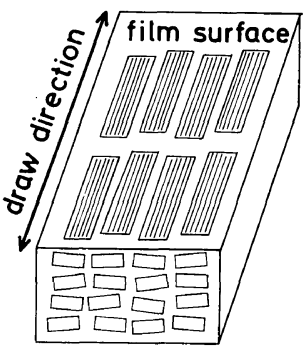

drawn film
Figure 6. Schematic models for the orientation of crystallites in the original and drawn film. Each rectangle represents the crystallites of polymer.

Table I. Photocurrent in PVK-TNF film along $X, Y$, and $Z$ directions ${ }^{\mathrm{a}}$

\begin{tabular}{|c|c|c|c|}
\hline \multirow{2}{*}{$\frac{\text { Light source }}{\mathrm{nm}}$} & \multicolumn{3}{|c|}{ Photocurrent $/ \mathrm{A} \mathrm{cm}^{-2}$} \\
\hline & $J_{X}$ & $J_{Y}$ & $J_{Z}$ \\
\hline 400 (surface) & $1.6 \times 10^{-8}$ & $0.4 \times 10^{-8}$ & $1.6 \times 10^{-8}$ \\
\hline 620 (bulk) & $8.8 \times 10^{-9}$ & $7.8 \times 10^{-9}$ & $8.8 \times 10^{-9}$ \\
\hline
\end{tabular}

a Thickness, $22 \mu \mathrm{m}$; temp, $344 \mathrm{~K}$.

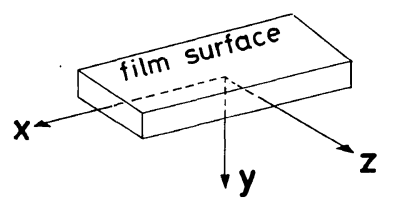

Figure 7. Definition of the three orthogonal axes.

(=6.4) as pointed out by Hirota. ${ }^{4}$ These results clearly demonstrated the validity of our analyses as well as the precision of our measuring system.

ii) Anisotropy of Photocurrent in the Oriented PEN Films

Figure 8 shows the relationship between photocurrent in the three orthogonal directions and the draw ratio. The definition of the axes is shown in Figure 9. The $X$ axis is parallel to the drawing direction, the $Z$ axis is perpendicular to the $X$ axis within the film surface, and the $Y$ axis is perpendicular to the film surface. Both $J_{x}$ and $J_{z}$ increased with the 


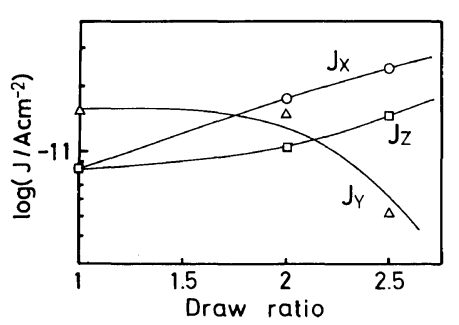

Figure 8. Relationship between photocurrent in three orthogonal directions and draw ratio.

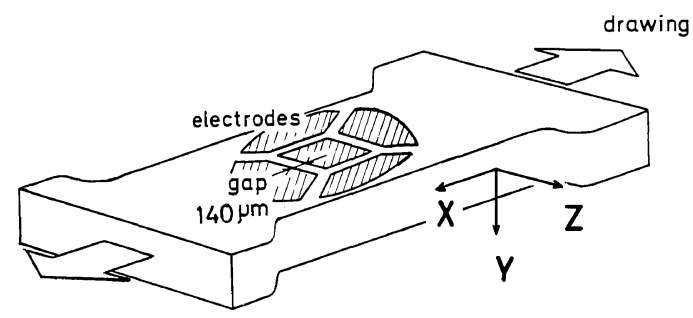

Figure 9. Definition of the'three orthogonal axes.

increase of draw ratio, whereas $J_{y}$ decreased. As was shown in the previous section, the order of the orientation of crystallites along $X$ and $Z$ axes increased with the increase of the draw ratio, but that along $Y$ axis became worse with drawing. Therefore, one finds a clear correlation between the anisotropic value of the photocurrents and the degree of orientation of crystallites in the polymer films. Specifically, the decrease of $J_{y}$ with the increase of the draw ratio can be reduced to the resultant decrease of the crystallite orientation along the stacking direction of naphthalene rings.

The value of photocurrent $\left(J_{\mathrm{p}}\right)$ is generally given by the equation;

$$
J_{\mathrm{p}}=e \times \mu \times F \times \Delta n
$$

where $e$ is the electronic charge, $\mu$, the mobility of carriers, $F$, the electric field, and $\Delta n$, the number of photo-generated carriers. $\Delta n$ is given by

$$
\Delta n=G \times \tau \times L
$$

$G$ is the generation efficiency, $\tau$ is the life time of carriers and $L$ is the number of absorbed photons. Drawing of films may bring about the alteration of $\Delta n$ as the results of the change of their physical states. However, $\Delta n$ does not bear anisotropic nature at all. Consequently, the presence of the influence of higher-order structure on the anisotropy of photocurrents should be ascribed to the anisotropy in the carrier mobility. In other words, the orientation of crystallites in the film surely reflects on the transport of electronic carriers. The more the crystallites orient regularly, the faster electronic carriers can migrate.

Finally we stress that the anisotropy of electronic conduction in the oriented PEN film which has no conjugated system of $\pi$-electrons in a molecular chain was found.

Acknowledgment. We are indebted to Dr. S. Hirota (Electrotechnical Laboratory, Japan) for providing the masks of electrodes on evaporating gold. We also thank Dr. T. Tsutsui for his helpful discussions during the course of this study.

\section{REFERENCES}

1. D. A. Seanor, J. Polym. Sci., C, 17, 195 (1967).

2. Y. W. Park, M. A. Druy, C. K. Chiang, A. G. MacDiarmid, A. J. Heeger, and S. Ikeda, J. Polym. Sci., Polym. Lett. Ed., 17, 195 (1979).

3. W. Schermann and G. Wegner, Makromol. Chem., 175, 667 (1974).

4. S. Hirota, J. Appl. Phys., 50, 3003 (1979).

5. S. Hirota, J. Appl. Phys., 53, 3792 (1982).

6. H. J. Wintle and T. C. Chapman, J. Appl. Phys., 51, 3435 (1980).

7. Z. Menčik, Chemický Prǔmysl., 17, 78 (1967). 